\title{
La relación entre Bioética y Salud Pública
}

\author{
Claudia Patricia Tacumá Prada - Blanca Elpidia Tovar Riveros
}

\begin{abstract}
Resumen: el proceso de toma de decisiones desde la Salud Pública (SP) se basa en el ser y el deber ser. En Colombia los comités de bioética, ética de la investigación y ética clínica han funcionado con sus propios estatutos para garantizar el derecho a la salud, a pesar de las reformas a la seguridad social. Los antecedentes los llevaron a una fragmentación del sistema de salud caracterizada por la axiología neoliberal (economía de mercado según capacidad de pago) y normativa del Estado Social de Derecho. El sistema pretende resolver esta fragmentación, pero existen problemas estructurales del mercado que solo se solucionarían si funcionaran los mecanismos de regulación y el papel del Estado. Es decir, el sistema es insuficiente para lograr satisfacer las necesidades de la salud debido a las interacciones entre actores sociopolíticos. Este proceso conduce a opciones utilitaristas que sustentan la tendencia en temas de investigación. Los valores en SP junto con la ética de la SP son variables. Para la ética de la SP los valores de la práctica son de importancia pública, relacionados con los objetivos de la sP y los profesionales.
\end{abstract}

Palabras clave: bioética; comités de bioética; comités de ética; salud pública

Recibido: 20/07/2020

Aceptado: 06/08/2021

Disponible en línea: 31/12/2021.

Cómo citar: Tacumá Prada CP, Tovar Riveros BE. La relación entre Bioética y Salud Pública. Rev. latinoam. bioet [Internet]. 31 de diciembre de 2021 [citado 31 de diciembre de 2021];21(2):143-56. Disponible en: https://revistas.unimilitar.edu.co/index.php/rlbi/article/view/5020

a Máster en Administración de salud. Candidata a doctora en Salud Pública. Universidad de Ciencias Aplicadas y Ambientales. Bogotá, Colombia. Correo electrónico: ctacuma@udca.edu.co, ORCID: https://orcid.org/0000-0001-6235-382X

b Magister en Educación y Docencia Universitaria, Candidata a doctora en Bioética. Fundación Universitaria del Área Andina. Bogotá, Colombia. Correo electrónico: btovar@areandina.edu.co, ORCID: https://orcid.org/0000-0003-4784-3163 


\section{The relationship between Bioethics and Public Health}

Abstract: Public Health (PH) decision-making is based on what is and the ought-to-be. In Colombia, the bioethics, research ethics, and clinical ethics committees have operated with their own bylaws to guarantee the right to health, despite social security reforms. The antecedents resulted in a fragmented health system characterized by neoliberal axiology (market economy according to the ability to pay) and the regulations of a Social State under the rule of law. The system tries to solve this fragmentation, but the structural problems in the market would only be overcome if the regulatory mechanisms and the role of the State worked. In other words, the system is insufficient to meet health needs due to interactions between socio-political agents. This process leads to utilitarian choices that sustain the trend in research topics. PH values, along with PH ethics, are variable. For PH ethics, practice values related to PH objectives and professionals are of public importance.

Keywords: Bioethics; bioethics committees; ethics committees; public health

\section{A relação entre Bioética e Saúde Pública}

Resumo: O processo de tomada de decisões no contexto da saúde pública (SP) se baseia no ser e no dever ser. Na Colômbia, os comitês de bioética, ética da pesquisa e ética clínica vêm funcionando com seus estatutos para garantir o direito à saúde, apesar das reformas à seguridade social. Os antecedentes os levaram a uma fragmentação do sistema de saúde caracterizada pela axiologia neoliberal (economia de mercado segundo capacidade de pagamento) e normativa do Estado social de Direito. O sistema pretende resolver essa fragmentação, mas existem problemas estruturais do mercado que somente seriam solucionados se funcionassem os mecanismos de regulamentação e o papel do Estado. Isto é, o sistema é insuficiente para satisfazer as necessidades da saúde devido às interações entre atores sociopolíticos. Esse processo conduz a opções utilitaristas que apoiam a tendência em temas de pesquisa. Os valores em SP junto com a técnica da SP são variáveis. Para a ética da SP, OS valores da prática são de importância pública, relacionados com os objetivos da SP e dos profissionais.

Palavras-chave: bioética; comitês de bioética; comitês de ética; saúde pública 


\section{Introducción}

Este artículo reflexiona sobre la bioética, ética de la salud pública (SP) y el desarrollo de la investigación para la toma de decisiones que se basa en el "ser", el "deber ser" y la democracia. Esta dicotomía permite que exista una pluralidad de modelos teóricos, paradigmas y disciplinas involucradas en el análisis de los determinantes alrededor de un mismo objeto complejo de análisis: el proceso de la salud y la enfermedad. Para lograr los derechos sociales, económicos y culturales que resultan de las relaciones entre los sistemas políticos y económicos son determinantes las opciones colectivas resultantes. Estas se articulan a largo plazo en la relación de Estado-sociedad.

Lo primero de lo que se debe ocupar la sp son en los conflictos que surgen en los comités de bioética CBE. Un gran aporte para el desarrollo de la democracia debe partir de dos formas de interpretación. La primera está dirigida a los ideales y la segunda a los hechos. Por otra parte, la sP es el sistema democrático ubicado en una deontología que, de acuerdo a Bentham citado por Sartori (1), se utiliza como sinónimo de "ciencia de la moralidad". En ese sentido, en la sP se deducen oraciones normativas a partir de oraciones prescriptivas; aquellas que dicen lo que "debe ser". Satori también menciona que la democracia, a diferencia de la aristocracia, es una estructura social horizontal en lugar de vertical. Es decir, que debe integrar a los involucrados y no solo los entes reguladores y el Estado (1).

Además, el autor sostiene que la fuente de la reflexión, la crítica y las decisiones es el conocimiento de las distintas opciones que les son accesibles al ser humano (1). De ahí la necesidad de trabajar en las relaciones de poder. Desde la postura de Foucault (2) la relación entre el sujeto y el poder no es exclusiva a las parejas, sino que se puede evidenciar en todas las acciones individuales y colectivas. El poder solo se ejerce cuando este se ejecuta como acción. Por lo que no debe evolucionar de la dominación a la emancipación, como se explica en la figura 1 (3).

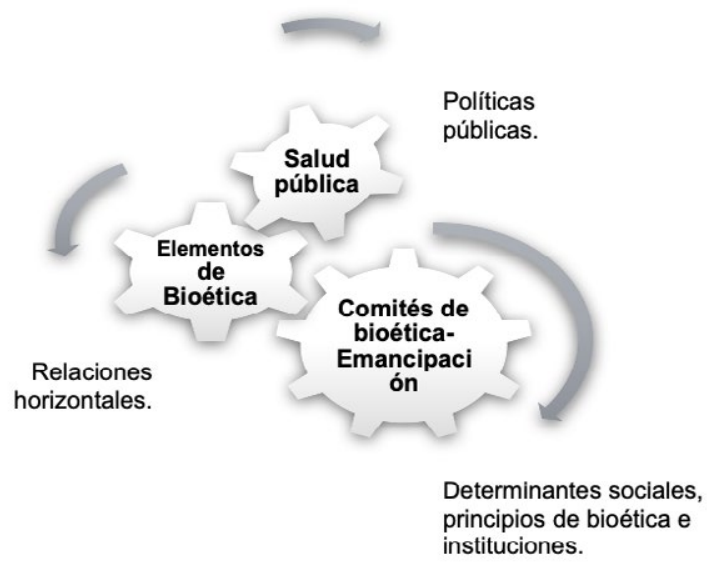

Figura 1. Relación entre bioética y SP.

Fuente: elaboración propia. 
Se argumenta que el mercado logra otorgar un derecho que resulta insuficiente para las necesidades de la salud colombiana. Esto debido a las presiones de ofertas e incentivos de investigación de sus actores y de sus patrocinadores; quienes tienen la intención de implementar protocolos atractivos para aquellos que encuentran en los países latinoamericanos una fuente de sujetos poco empoderados de sus derechos, y un sin número de comités que se benefician de las falencias de la sP.

Por tal motivo, algunos países han implementado normas y mecanismos de evaluación para la investigación en salud, con el fin de ajustarla a la realidad local. Desde esta perspectiva parte la hipótesis de que existe un enfoque transdisciplinario que es conmensurable entre la sp y la bioética. Este está sustentado en dos abordajes: el primero es la naturaleza y estructura general epistemológica de la bioética y la SP. Estos últimos dialogan por el bienestar de la persona. Mientras el segundo aborda los problemas de metodología de la bioética y la SP que se relacionan estrechamente con la biotecnología. La anterior está centrada en los avances científicos que convergen en la búsqueda del bienestar de la persona que requiere mantener, mejorar o rehabilitar el proceso de salud.

\section{La naturaleza y estructura general epistemológica de la SP y la bioética.}

La SP inicia en el periodo de posguerra (1945-1991) (4). Pero, anterior a ella, durante el siglo XIX, sucedió el impacto del capitalismo con las raíces sociales de la enfermedad en Europa. Estas raíces estaban relacionadas, a su vez, con los orígenes de la medicina social. En 1840, Engels F (5), se centró en el estudio del sistema político-económico y el capitalismo industrial con la estructura de clases para demostrar que las tasas de mortalidad eran inversamente proporcionales a la clase social. Posteriormente, a lo largo del siglo XX, existieron componentes históricos entre la SP y los servicios de salud que llevaron a el análisis de la relación de los sistemas políticos y económicos subyacentes. Lo que explica el sentido, los alcances y las limitaciones de las reformas.

Durante la década de 1930, Allende S expuso la importancia de la medicina social latinoamericana en un contexto geopolítico condicionado por los problemas de salud de la población chilena; derivados a su vez por las condiciones políticas y económicas del país. Para 1948, Wirchow (4) desarrolló la teoría de las epidemias y las estructuras político-económicas que promovía la propagación de las condiciones materiales de vida en las que aparecían las enfermedades. Así, se descubrió que las condiciones determinantes como el salario, la nutrición, el impacto de la inflación, las condiciones sanitarias y de vivienda precarias, las enfermedades de transmisión sexual, el alcoholismo o las leyes inadecuadas explicaban un efecto profundo en la morbimortalidad temprana (4).

Con las altas tasas de morbimortalidad temprana surge el desarrollo y la comercialización de productos y avances tecnológicos que fortalecieron el sistema capitalista y su expansión (4). Sin embargo, cuando un nuevo producto satura el mercado, la tasa de ganancias baja y surgen estrategias como la búsqueda de nuevos mercados vía exportación internacional. Sin importar las consecuencias de los mismos, y solo por una visión de los sistemas políticos y económicos hacia su necesidad.

A finales de los años sesenta y principios de los setenta, surge la bioética en respuesta a los dilemas éticos emergentes en la salud. Esta se centró en los dilemas generados por la medicina de alta tecnología y los problemas éticos de programas y regulaciones de SP, más que en cuestiones de salud poblacional. Además, simbolizó la importancia del profesionalismo médico más allá de la relación médico-sujeto de cuidado. No obstante, se le cuestionó la ponderación de su orientación individualista, basada en los principios de autonomía, beneficencia, justicia y no-maleficencia para el ser humano, sobre el interés general de la ciencia y la sociedad (6). El cual es importante por la salud de la población y el enfoque poblacional y social de la sP (4).

Estos dilemas éticos que se proyectan en la bioética son de tal complejidad y repercusión social que, por la responsabilidad de los modelos de comportamiento que se involucran en la sociedad, no pueden dejarse al arbitraje de los científicos y 
de los profesionales. Por el contrario, deben sustentarse en un enfoque transdisciplinar. De acuerdo a Kottow (7), la bioética se valida "en la medida que logra insertarse en la realidad cultural de su sociedad y en el campo de deliberación que la requiere"(p. 286). Es decir, la bioética es válida en la medida en la que atienda la especificidad del quehacer de la SP y sus fundamentos teóricos.

Asimismo, se pueden presentar conflictos cuando hay un riesgo sustancial de que los intereses secundarios de una o varias partes interesadas en una investigación incidan de forma indebida sobre su juicio. Comprometiendo así el objetivo primario de la investigación por el ambiente competitivo de los investigadores ${ }^{1}$ (9). Incluso, de acuerdo a las recomendaciones de la Unesco $(10,11)$, para evitar el fracaso se debe solucionar la falta de consenso en los asuntos de procedimiento como: ¿a quién deben servir los comités?, ¿quiénes deben integrar los comités?, ¿qué temas deben tratar?, ¿cuál es el alcance de su autoridad?, ¿cuál es la formación que deben tener?

La bioética como concepto nace en 1927 con Fritz Jahr, teólogo, filósofo y profesor alemán. Él utilizó el término por primera vez con el fin de resolver la relación entre las formas vivas de la realidad, para ayudar a la ciencia y la tecnología a seguir un camino de responsabilidad moral y de conducta hacia el respeto de todas las formas de vida $(12,13)$. Lo anterior estaba determinado por la rigurosidad y categórica exigencia de hacer elecciones morales pragmáticas y prudentes, y por el concepto imperativo de "respetar a cada ser viviente desde un principio" (12, p. 20).

Posteriormente, en 1970, Van Potter, bioquímico y oncólogo, acuña el término y la define como "Bioética: ciencia de la supervivencia" (14, p. 589). En los años setenta, también se crearon los primeros centros de bioética en E.U. y las comisiones de ética que utilizaron como enfoque el principialismo. Este se basa en la resolución de problemas bioéticos a la aplicación de principios. Así, surgió la primera enciclopedia de bioética y el Informe Belmont (15) y, consecuentemente, se establecieron los lineamientos básicos de las instituciones, como lo son los CBE.
El vocablo bios (vida) representaría a las ciencias biológicas, y ethos (ética) a la definición de los valores y normas de conducta; la bondad o la maldad de los actos humanos y las reglas de comportamiento (16). Para Hottois (15), "la bioética cubre un conjunto de investigaciones, de discurso de prácticas, generalmente pluridisciplinarias y pluralistas, que tienen como objeto aclarar y si es posible, resolver preguntas de tipo ético, suscitadas por la I y D biomédicos y biotecnológicos en el seno de sociedades caracterizadas, en diversos grados por ser individualistas, multiculturales $y$ evolutivas" (p. 26).

Con el propósito de su aplicación surgen los problemas metodológicos creados por la I y D tecnocientíficas, sumado a los desacuerdos que surgen en todos los comités de bioética; como el gran aporte para el desarrollo de la democracia, los desacuerdos interdisciplinarios y los intereses que se derivan de ellos. Por esta razón, Hottois, propone una nueva metodología para que las instituciones puedan lograr acuerdos mínimos, pragmáticos y de acuerdo al tamaño de los conflictos expuestos. Además, establece que la misión de los CBE es "aclarar un tema desde el punto de vista de los valores y de las normas en el seno de una sociedad o de un conjunto de naciones". Siempre y cuando se eviten los consensos "forzados" y los disensos "perezosos"(15, p. 41). El autor (13) reconoce también que "no se puede separar la acción individual y colectiva basada en valores y normas". Puesto que el conocimiento de leyes y hechos "científicamente establecidos [...] dan luz sobre las condiciones y consecuencias" (18, p. 66).

Los CBE se definen entonces como grupos interdisciplinarios cuyas funciones son la educación, difusión, promoción y defensa de la bioética en las instituciones de salud. Estas funciones las desempeñan a través de actividades educativas, consultivas y normativas que facilitan la relación más racional entre la persona humana, la biotecnología y el medio ambiente (15, p. 35). Por tal razón, en momentos de crisis o pandemias la normativa de aplicación en cada caso debe ser autorizada previamente por los comités de ética de la investigación que disponga. Se debe asumir la responsabilidad forzada porque cabe una interpretación mecánica 
de las normas. Aunque ello no implica una interpretación inflexible que impida el interés colectivo, sin detrimento de la dignidad y del derecho a la intimidad del individuo (19).

Los inicios de la bioética se centran en el análisis de los conflictos que surgen en la práctica clínica. Por ello se da en el sistema de salud, el es más frecuente en la vida de las personas. En ese sentido, es mejor percibida y comprendida. Diferente del Estado que presta otro tipo de atención a los servicios sanitarios públicos, frente a la atención médica personal. Por eso, cuando se produce un debate público sobre la atención sanitaria y sus reformas, interviene el cuidado de la salud personal, los centros de salud y sus capacidades. Sin embargo, no existe un alto grado de conciencia pública en esta área. Un ciudadano común puede no conocer sobre la sp y su definición clásica:

la ciencia y el arte de prevenir la enfermedad, prolongar la vida y promover la salud y la eficiencia a través de la organización de la comunidad para el saneamiento del medio ambiente, el control de las infecciones transmisibles, la educación del individuo, la organización de servicios médicos y de enfermería para el diagnóstico temprano y la prevención y el tratamiento de la enfermedad y el desarrollo de la maquinaria social para asegurar un nivel de vida adecuado para el mantenimiento de la salud, para permitir a cada ciudadano el derecho de nacimiento de salud y longevidad (20, p. 30)

Por su parte, Cortina define la bioética como "el estudio sistemático de las dimensiones morales, incluyendo la visión moral, decisiones, conducta y políticas, de las ciencias de la vida y la atención de la salud, empleando una variedad de metodologías éticas en un contexto interdisciplinario" (20, p. 4). En Latinoamérica, la bioética de Cortina se presenta con unos rasgos comunes: las experiencias sociales, culturales y políticas diferentes a las de E.U. En ese sentido, no se reduce al bios tecnológico y al ethos liberal. Por el contrario, hace énfasis en el bios "humano" y el ethos "comunitario" que se deriva en la preocupación de la justicia local y global, las políticas públicas y la intervención en los contextos concretos (20).

\section{Los problemas de metodología de la bioética y SP ligados a la biotecnología.}

La salud de las poblaciones es una ocupación de la sp y medida socioeconómica, a diferencia de los avances en las condiciones biomédicas. Si bien es cierto que la SP requiere de la biomedicina para su trabajo, para la detección temprana de enfermedades y las técnicas que requieren los programas, la salud plantea una serie de problemas morales. Estos problemas van más allá de los anteriores límites de la bioética y requieren su propia forma de análisis ético $(4,7)$. Sin embargo, existe escasa literatura que aporte marcos conceptuales sobre la ética de la salud pública y sobre los criterios en cuanto a la toma de decisiones.

La aplicación masiva de políticas públicas ha permitido cambios en el mercado de trabajo, junto con redes insuficientes de servicios sociales que no admitieron considerar las características propias de cada país. Esto dio paso a la fragmentación de servicios de salud ${ }^{3}$, promoviendo una asignación ineficiente de los recursos en los contextos como un todo $(21,22)$. El principialismo se ha convertido en el enfoque predominante de la bioética a través de ciertos contextos socioculturales. Mientras que las teorías utilitaristas influyen en muchos espacios, incluidos los sistemas de salud. Siguiendo la lógica utilitarista, la distribución debe generar la máxima satisfacción al mayor número de personas con el menor costo posible. En ese orden de ideas, un ejemplo de aplicación de esta lógica en los sistemas de salud podría ser la reforma de la seguridad social en Colombia (23). Por lo que este modelo económico y sus consecuencias son el principal factor de análisis.

Con respecto a las normas para la investigación epidemiológica y la investigación biomédica que involucra sujetos humanos, en 1991 el cioms mencionó que, si las actividades de la práctica de la sP y la investigación biomédica se realizan simultáneamente, pueden resultar confusas (24). Igualmente, reconoció que la discusión entre "pacientes y sujetos individuales" no fue suficiente para estudios que incluían "grupos". En ese momento, el 
CIOMs realizó un consenso que reconoció que la aplicación en el contexto epidemiológico requería flexibilidad. Por esa razón, se podía omitir el consentimiento informado cuando el riesgo fuera mayor que el mínimo y la obtención del consentimiento hiciera la investigación impracticable.

La investigación epidemiológica no es simplemente otra forma de investigación, pues en la vigilancia una comunidad es el tema de preocupación, y esa vigilancia es una responsabilidad de la sP. Además, la misma organización describió la vigilancia en situaciones de brotes de emergencia. En estas situaciones extremas, la vigilancia no puede esperar la formalidad (25). De acuerdo a Coughlin (26), es necesario generar una ética de la SP, entendiendo como tal la identificación, el análisis y la resolución de los problemas éticos originados en la práctica y la investigación en sP. Schramm y Kottow $(26,27,28,29)$ resaltan que las especificidades de la ética en la sp que habían utilizado los principios clásicos de la bioética tienen una utilidad limitada. Puesto que la sp requiere de principios propios que se adapten mejor a ella.

Para debatir esos dilemas, la Unesco propuso cuatro tipos de comités de bioética: comité normativo y/o consultivo, comité de asociación de profesionales de la salud, comité de ética asistencial/ hospitalaria y comité de ética de la investigación. Los dos primeros no admiten confusiones porque tienen como función asesorar a funcionarios públicos e influir en la adopción de políticas científicas y de salud de ámbito nacional. Los de asociaciones de profesionales se ocupan de cuestiones de bioética planteadas por profesionales asistenciales. Su objetivo es debatir cuestiones de atención en prácticas clínicas centradas en el paciente. En contraposición, los comités de ética de la investigación abordan las cualidades científicas, el riesgo al que se exponen los participantes y los posibles beneficios para futuros pacientes que no participan como sujetos en ensayos clínicos. Los dos últimos comités tienen tendencia a combinar las dos funciones (11).

La Unesco es la directriz bioética internacional de la investigación en seres humanos, todo lo que es vida o tiene estrecha relación con la salud humana, animal, biótica y no biótica. El cumplimiento de lo dictado por esta organización está bajo la responsabilidad del gobierno desde lo nacional, regional y gestión del riesgo, ciencias de la vida y tecnología (30). En la Conferencia General de la onu para la Educación, la Ciencia y la Cultura, con la aprobación de la Declaración Universal sobre Bioética y Derechos Humanos, se definieron los quince principios de la bioética ${ }^{4}$. Sin embargo, la definición de la Unesco es demasiado amplia y admite una diversidad de funciones de los comités de ética asistencial (grupo de personas que aborda sistemáticamente las dimensiones éticas de las ciencias de la salud, las ciencias biomédicas, las políticas de salud y los casos clínicos complejos) (11).

Es cierto que existe una brecha entre la cantidad de situaciones con problemas éticos para la toma de decisiones y el escaso número de casos clínicos que se presenta a los comités. Una solución que toman algunos comités es designar a uno o más de sus integrantes como consultores individuales, o bien analizar los casos en subcomités de dos o tres integrantes. Pero no hay un acuerdo en cuanto a los consultores ético clínicos.

La experiencia tiene origen en Norteamérica y ha sido discutida por diferentes países. La consulta de ética clínica (CEC) se ha convertido en una norma de atención, pues es un mecanismo de apoyo a los pacientes, la familia y el personal durante situaciones éticamente difíciles en entornos clínicos. Aunque aún no hay reportes de su eficacia, estas CEC pueden ser realizadas por un comité de ética, un grupo de consultores de ética o un consultor individual, y con frecuencia se centra en los conflictos de atención percibidos por los pacientes, las familias o los proveedores de atención de salud. El servicio de CEC proporciona asesoramiento, información o mediación con recomendaciones $(31,32)$.

De acuerdo con The Nuffield Council on Bioethics (29), una ética de protección garante del Estado rector propone que cuanto más intrusiva sea una medida de salud pública, más fuerte deberá ser su justificación planteando una "escalera de intervención”. Además, la contribución de los derechos humanos y la ética en salud pública de Nixon y Fornam facilitan la justificación de intervenciones más sólidas. Enfatizando la obligación de los estados de mejorar la salud gradualmente para 
reconocer así los derechos humanos, y reorientando los esfuerzos sobre salud y enfermedad entre poblaciones y personas marginadas.

El Consejo Nórdico de Ministros, plantea tres características de problemas que pueden surgir como conflictos de la ética de la SP(34):

Los conflictos de libertad individual y la sP. Dentro de ellos, la ética del control de las infecciones, tamizajes poblacionales (genéticos, prenatal, poblacional) y el desafío de la selección de la población. (34. p. 11-47)

La responsabilidad de la salud y estilos de vida que contiene la SP, cambio climático, ética y la obesidad planteada como una enfermedad personal o un problema público.(34, p. 61-107)

La igualdad y justicia social que contiene los determinantes sociales y la salud de las poblaciones y grupos de población vulnerables, así como el establecimiento de prioridades o la discriminación étnica y de salud en las zonas de asentamientos. (34, p. 119-147)

Además, el Consejo Nórdico de Ministros también señala el riesgo de estigmatización de las personas cuyos hábitos se consideran no saludables (34). Por ello, la práctica de la sp debe estar respaldada por el Estado. Diferente al rol de investigación en sp que depende de la voluntad de las personas para adquirir los datos. En ese sentido, un rasgo distintivo de la sP es la responsabilidad de la sociedad en su conjunto, así como la búsqueda de respuestas a problemas de salud que no son solamente resueltos por la asistencia médica. El concepto de lo público se centra en las relaciones interpersonales que permiten el surgimiento de nuevos enfoques para la ética de la sp $(7,34,35)$.

En el 2013, el cıoms implementó las recomendaciones sobre la ética de prevención, atención y control de la tuberculosis (36). En el 2015, se implementó el manual de entrenamiento sobre ética en epidemias, emergencias y desastres (37), la guía para el manejo de problemas éticos en manejos de brotes de enfermedades infecciosas (38), y en el 2017, la guía de la oms sobre problemas éticos de la vigilancia en salud pública (24). Estos manuales sirven para aclarar las actividades realizadas por autoridades sanitarias y por otros fuera de ellas. En las investigaciones que no surgen de la autoridad sanitaria, rigen los requisitos de una investigación en salud. Los diferentes, nuevos y complejos dilemas de las ciencias biológicas requieren una formación más especializada de los responsables de las evaluaciones éticas de los protocolos de investigación. Existen varios métodos para el análisis de los protocolos de investigación como requisitos básicos para la investigación clínica:
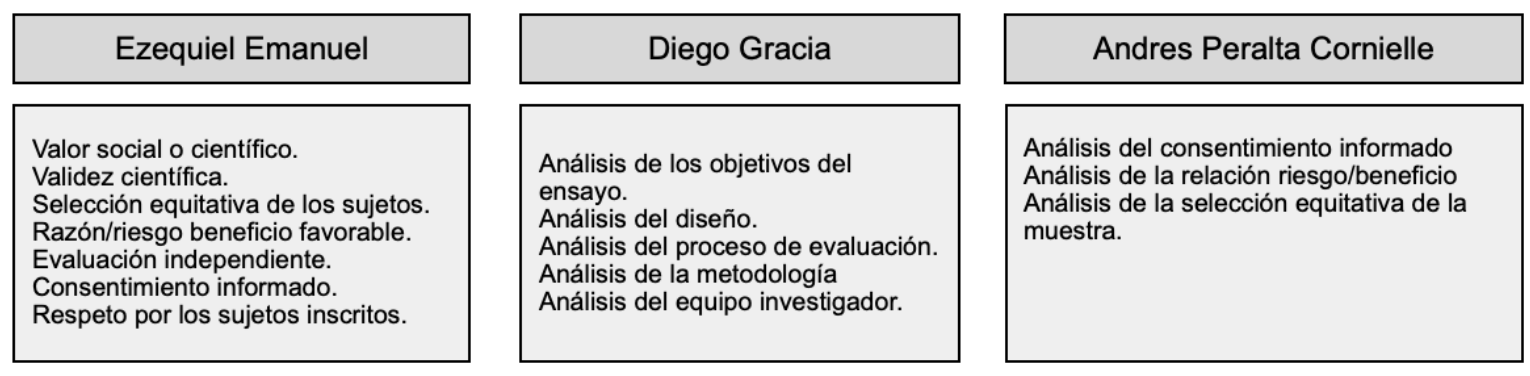

Figura 2. Proceso metodológico para investigadores.

Fuente: elaboración propia. 
Kottow (7) propone que "Latinoamérica sólo tendrá algún poder si cohesionan sus fuerzas, una necesidad que fluye del momento de una bioética regional que comparta no sólo problemas, sino que proponga y apoye también programas de acción resolutiva" (p. 119). Además, plantea las diferencias entre la investigación en SP y la práctica de sp. Para él, la primera requiere de los requisitos de la ética de investigación: minimizar los riesgos, brindar beneficios a los participantes y evitar e informar sobre conflictos de interés. Asimismo, está la obligación desde la epidemiología respecto a la investigación en comunidades, desarrollo y formas del consentimiento informado, custodia de la privacidad, la confidencialidad y cómo se estructuran y funcionan los comités de ética de la investigación (7). Es decir, las mismas normas de otros campos.

La segunda, por su parte, es una responsabilidad de la autoridad sanitaria. Por lo que en la práctica de la sp se debe diferenciar entre vigilancia epidemiológica, organización de la respuesta a emergencias, respuestas y evaluación de programas y la forma en que estas actividades se vinculan. Es por todo lo anterior que las consideraciones bioéticas prevalecen sobre los modelos científicos de decisión en la formulación de políticas públicas en cada país (39). En el caso de Colombia, la tamización neonatal requiere normas de mayor jerarquía articuladas con las actividades de la atención en salud y los principios bioéticos.

Los estudios predicen que una de las bases sólidas de la sp es la investigación para el desarrollo de las políticas y prácticas de SP (40). En consonancia, el personal de SP y los representantes de los comités de ética en su práctica deben trabajar en una sola dirección. Partiendo de lineamientos para la identificación y valoración de las sociedades, caracterizadas en diversos grados desde la individualidad, la multiculturalidad y la evolución (35).

En Colombia ha funcionado con anterioridad a la Comisión Intersectorial de Bioética (СIB), el Tribunal Nacional de Ética Médica como órgano consultivo y asesor del gobierno para el estudio, análisis y formulación de políticas públicas en la protección del ser humano. Tan solo en 1991, por norma, fueron constituidos los comités de ética hospitalaria mediante la Resolución 13437 de 1991(41) para ser funcionales en las Entidades Prestadoras de Servicios de Salud (EPS) del sector público y privado. Más adelante, surgió el Decreto 1757 de 1994 como una forma de participación social institucional ejercida mediante los comités de ética médica (42). Sin embargo, en el 2007 la Ley 1164 denominó a los comités de ética y bioética como organismos de apoyo para el desarrollo del talento humano en salud (43). Dejando de lado la distinción de su significado y dificultando su operación práctica (44).

La ley 1374 creó el Consejo Nacional de Bioética (CNB) en el 2010 (18), y en el 2019 se estableció la normativa para conformar los integrantes y funciones de dicho CNB para establecer un diálogo interdisciplinario. Donde una de sus funciones es conceptuar y asesorar en la reglamentación los diferentes aspectos de la bioética ocasionados por el avance de las ciencias y la tecnología (46). Esta función estaba ausente en la resolución del Ministerio de Salud (41), única norma orientadora sobre investigación en seres humanos.

Por consiguiente, la sP se comenzó a entender como un sector del Estado que se encargaba de la salud colectiva. Donde, paralelamente, la bioética y su intervención con los comités de bioética se relacionaban estrechamente. Los comités asistenciales y generales estaban asignados de forma individual. Mientras que los comités de bioética se asignaron de forma colectiva. Reafirmando así que la relación entre bioética y salud pública es un campo de saberes, prácticas y metodologías que conlleva a reflexionar sobre las condiciones necesarias para una buena decisión con un enfoque interdisciplinario.

En Colombia, se logró la estandarización del funcionamiento de los diversos comités a nivel institucional, local y regional. Además, se logró un registro unificado de los comités o comisiones de bioética (desde ahora CBE) y servicios cómo las consultas de ética asistencial. No obstante, aún existe una falta de estandarización de las políticas de los mismos. Lo que a su vez se resume poca colaboración de la dirección, falta de sensibilidad con 
el tema y desconocimiento de la labor por parte de los participantes (47).

Es allí donde los CBE deben brindan su mayor aporte, dado que la preocupación general por el paciente individual que se encuentra en la ética clínica no es parecida a la preocupación por la salud de la población. De hecho, para Upshur (48) el modelo de la ética hospitalaria es poco apropiado por el tipo de razonamiento para la ética de la salud pública. En efecto, la frontera entre lo individual (ética asistencial y ética de la investigación) y lo colectivo (comités de bioética) está ahí, pero también se deben replantear los problemas como conjunto social y no individual.

Upshur (48) retoma cuatro principios para la justificación de la intervención de salud pública: "principio de daño", creado por Mill Stuart, donde el único poder que se puede ejercer sobre cualquier miembro de una comunidad es para evitar daño a los demás. "Principio de los medios menos restrictivos o coercitivos", que reconoce que existe una cantidad de medios para lograr fines de salud pública y toda fuerza de poder del Estado debe reservarse para circunstancias excepcionales. "Principio de reciprocidad", el cual indica que una vez que se justifica la acción de salud pública, existe una obligación de una entidad social de ayudar a las personas a cumplir sus obligaciones éticas. Por último, el "principio de transparencia" que establece que todos los interesados legítimos deben participar en el proceso de toma de decisiones y tener la misma participación en las deliberaciones, con responsabilidad y claridad. Bajo esta lógica, la metodología para nuevos comités de bioética debe ser retomar la democracia social como el conjunto de las democracias primarias determinadas por pequeñas comunidades y asociaciones voluntarias concretas que alimentan a la democracia hacia la base (2). De esta forma, se involucra a todos en las nuevas instituciones. Estos aspectos metodológicos se presentan en la figura 3.

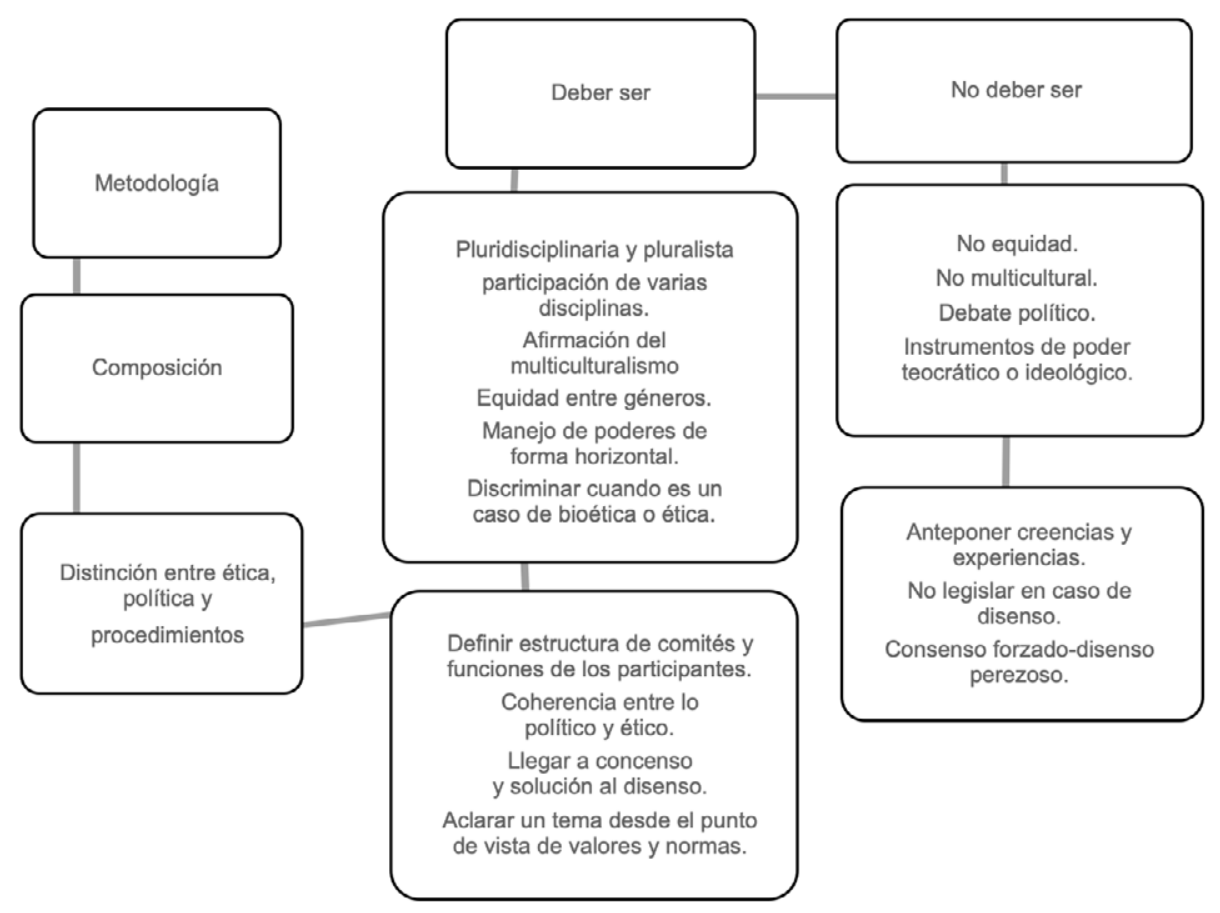

Figura 3. Aspectos claves de la metodología de los comités de bioética.

Fuente: elaboración propia a partir de los lineamientos de los comités de bioética (20). 


\section{Conclusión}

La perspectiva académica y política entre bioética y salud pública permite identificar dilemas éticos propios de la SP, basándose en los aspectos claves de la metodología de los comités de bioética. En ese sentido, las condiciones concretas de la práctica de la bioética han cambiado desde su concepción. No obstante, todavía se requiere con urgencia una nueva metodología y el logro de acuerdos mínimos, pragmáticos, que correspondan al tamaño de los conflictos expuestos. Retomando a Hottois, los comités de bioética "buscan aclarar un tema desde el punto de vista de los valores y de las normas en el seno de una sociedad o de un conjunto de naciones", siempre y cuando se eviten los consensos "forzados" y los disensos "perezosos" (15, p. 41).

En salud pública, se expresan e interactúan complicaciones de la cuarta revolución industrial que involucran sociedades e individuos. Sin embargo, la toma de decisiones no puede perder de vista esta complejidad para simplificar problemas entre bioética y salud pública. Por ende, se concluye que las disciplinas son conmensurables, y por tanto el diálogo interdisciplinar permite conocer el nivel de desarrollo de estas disciplinas y su reflexiva e importante postura en la participación de los diversos comités en Colombia. Es evidente que hay brechas en las disciplinas, pero también es importante reconocer que partiendo de las relaciones de poder existentes y el manejo de la democracia es posible encontrar un camino convergente.

Desde la mirada de Stigliz, premio nobel de economía, para que ocurran cambios en las instituciones, el Estado y la sociedad, los países deben modificar sus sistemas y generar políticas que beneficien al colectivo y no a particulares. Para ello, una posibilidad es la de crear nuevamente confianza. Dar el valor que merecen las personas para construir de nuevo una democracia sólida que controle y equilibre el poder político de la riqueza concentrada en unos pocos. De igual manera, se debe permitir que todos tengan acceso al conocimiento para que puedan exigir una adecuada redistribución de recursos. Desde la bioética la función es velar por la creación de políticas públicas que beneficien a todos. Es decir, el propósito debe ser la equidad y la justicia para todos, para así disminuir la brecha en lo social, lo político y lo económico. Asimismo, se debe fortalecer la confianza de las personas; reconstruir el tejido social para armonizar los modos de vida, empoderando a las personas desde el respeto hacia la autonomía, la libertad, la beneficencia, la justicia, la solidaridad consigo mismos y los demás (22).

En relación, los comités de bioética y ética deben partir de la lectura comprensiva y puesta en práctica de las políticas públicas para trabajar en concordancia por el bien común. En ese orden de ideas, Noël Roth (48) argumenta que para la implementación de políticas públicas deben involucrarse a distintas instituciones y actores con evaluación que necesitan de un dispositivo más práctico. Es decir, pluralista desde varias perspectivas. En concordancia con la problemática actual de salud pública (pandemia COVID-19), las investigaciones deben tener en cuenta no sólo el diligenciamiento de la formalidad de documentos, sino también las dificultades psicosociales de los participantes; como lo son las emociones para saber qué hacer con la construcción de las situaciones de crisis (49). Se debe reconocer al otro para hacer deliberación de la situación y así comprender los contextos individuales y colectivos, con el fin de no vulnerar a las personas. El miedo no debe cohibir a ninguno de los actores involucrados (51).

\section{Referencias}

1. Sartori G. ¿Qué es la democracia? [Internet]. Ciudad de México: Tribunal Federal Electoral; 1993. [citado 2020 oct. 5]. Disponible en: https://archivos.juridicas.unam. $\mathrm{mx} / \mathrm{www} / \mathrm{bjv} / \mathrm{libros} / 3 / 1135 / 1 . p d f$

2. Álvarez-Sánchez Y. El poder y las relaciones de poder en las organizaciones: algunas aproximaciones teóricas desde las perspectivas de Michel Foucault, Pierre Bourdieu y Max Weber. Gest. Soc. [Internet]. 2011 [citado 2020 mzo. 5]; 4(1):145-61. Disponible en: https:// www.ses.unam.mx/docencia/2019I/Alvarez2010_ElPoderYLasRelacionesDePoder.pdf

3. Álvarez N. Poder y organizaciones: reflexiones desde Weber, Foucault, Luhmann y Bourdieu. Tendencias. [Internet]. 2019 [citado 2020 mzo. 5]; 20(1):226253. Disponible en: DoI: https://doi.org/10.22267/ rtend.192001.115 
4. Waitzkin H. Medicina y salud pública al final del imperio. Bogotá: Universidad Nacional de Colombia; 2013.

5. Rodríguez-Kuri A. México: guerra fría e historia política. Historia Mexicana [s. 1.]. [Internet]. 2016 [citado 2020 mzo. 23]; 66(2): 645-652. Disponible en: https:// search-ebscohost com.ezproxy.javeriana.edu.co/login.aspx?direct $=$ true $\& \mathrm{db}=\mathrm{edb} \& \mathrm{AN}=119758094 \&$ lan$\mathrm{g}=\mathrm{es} \&$ site $=$ eds-live

6. Fuchs C. Engels@200: Friedrich Engels and digital capitalism. How relevant are Engels's works 200 years after his birth? TripleC. [Internet]. 2020 [citado 2020 mzo. 6]; 19(1): 15-51. DoI: https://doi.org/10.31269/triplec.v19i1.1228

7. Council of Europe. Guide for research ethics committee members. 2013. Inst. Salud Carlos III [Internet]. 2012 [citado 2020 mzo. 5]; 1-61. Disponible en: https:// www.coe.int/t/dg3/healthbioethic/activities/02_biomedical_research_en/Guide/Guide_EN.pdf

8. Kottow M. Bioética en salud pública: Una mirada Latinoamericana. Editorial Universitaria.[Internet]. 2014 [citado 2020 mzo. 5]; 18(3): 286-289. Disponible en: http://www.scielo.br/pdf/csp/v32n10/1678-4464-csp32-10-e00114716.pdf

9. Callahan-Daniel JB. Ethics and public health: forging a strong relationship. Ethics public heal. [Internet]. 2002 [citado 2020 mzo. 5]; 92(2): 169-76. Disponible en: https://www.coe.int/t/dg3/healthbioethic/activities/02_ biomedical_research_en/Guide/Guide_EN.pdf

10. Organización Panamericana de Salud, Consejo de Organizaciones Internacionales de las Ciencias Médicas. Pautas éticas internacionales para la investigación relacionada con la salud con seres humanos. [Internet] Ginebra (Suiza): Consejo de Organizaciones Internacionales de las Ciencias Médicas (cioms); 2016 [citado 2020 mzo. 5]. Disponible en: https://cioms.ch/wp-content/uploads/2017/12/CIOMS-EthicalGuideline_SP_ INTERIOR-FINAL.pdf

11. Comité Internacional de Bioética de la unEsco. Responsabilidad social y salud. Informe del Comité de Bioética de la UNEsco [Internet]. Logroño (España): Organización de las Naciones Unidas para la Educación, la Ciencia y la Cultura (Unesco); 2018 [citado 2020 mzo. 5]. Recuperado a partir de: https://www. cibir.es/files/biblioteca/2018-UNESCO-Bioetica.pdf

12. Sass HM. El pensamiento bioético de Fritz Jahr 192719341. Aesthethika. [Internet]. 2011 [citado 2020 mzo. 5]; 6(2): 20-33. Disponible en: http://www.aesthethika. org/IMG/pdf/03_Sass_El_pensamiento_bioetico_ de_Fritz_Jahr.pdf
13. Núñez I. La bioética: un camino para el presente. [Internet] Guadalajara (México): Instituto Tecnológico y de Estudios Superiores de Occidente; 2017 [citado 2020 mzo. 10]. Disponible en: https://rei.iteso.mx/ bitstream/handle/11117/4680/Bioética.pdf?sequen$\mathrm{ce}=3$ \&isAllowed $=\mathrm{y}$

14. Muzur A, Rincic I, Sodeke S. The real Wisconsin Idea: the seven pillars of Van Rensselaer Potter's Bioethics. Journal of Agricultural \& environmental ethics. 2016; (29): pp 587-596. DoI: https://doi.org/10.1007/s10806016-9621-

15. Hottois G. ¿Qué es la bioética? Bogotá: Editorial Kimprés Ltda.; 2007.

16. Real Academia Española. Diccionario de la lengua española, 23 a ed., [versión 23.4 en línea]. Disponible en: https://dle.rae.es

17. Zanella DC. Humanidades e ciência: uma leitura a partir da bioética de Van Rensselaer (V. R.) Potter. Interface. [Internet]. 2018 [citado 2020 mzo. 10]; 22(65): 473-480. Disponible en: http://dx.doi.org.ezproxy.javeriana.edu.co/10.1590/1807-57622016.0914

18. Hottois G. Definir la bioética: retorno a los orígenes. [Internet] 2011; 6(2): 62-85. Disponible en: https:// www.redalyc.org/pdf/1892/189222558005.pdf

19. Winslow CEA. Changing challenges of public health. American J of Public Health. [Internet]. 1945 mzo. [citado 2020 abr. 5]; 35(3): 191-198. Disponible en: https:/ajph.aphapublications.org/doi/pdf/10.2105/ AJPH.35.3.191

20. Escobar J. Códigos, convenios y declaraciones de ética médica, enfermería y bioética. Bogotá: Ediciones El Bosque; 1998.

21. Comité de Bioética de España. Informe del Comité de Bioética de España sobre los requisitos ético-legales en la investigación con datos de salud y muestras biológicas en el marco de la pandemia covid-19 [Internet]. ; 2020. [citado 2020 abr. 1]. Recuperado a partir de: http://assets.comitedebioetica.es/files/documentacion/Informe\%20CBE-\%20Priorizacion\%20de $\% 20$ recursos\%20sanitarios-coronavirus\%20CBE.pdf

22. Cortina A. Bioética para el siglo XXI: construyendo esperanza. rib. [Internet]. 2016 my. 19 [citado 2020 mzo. 23]; (1): 1-12. Disponible en: https://revistas.comillas.edu/index.php/bioetica-revista-iberoamericana/article/view/6764

23. Iberoam Bioética [Internet]. 2016;(1):1-12. [citado 2020 abr. 2] DoI: https://doi.org/10.14422/rib.i01.y2016.001

24. Organización Panamericana de Salud. Las políticas públicas y los sistemas y servicios de salud. Salud en la 
Américas. 3. ${ }^{\mathrm{a}}$ ed. Washington: Organización Mundial de la Salud; 2011. p. 314-405.

25. Organización Panamericana de Salud, Organización Mundial de la Salud. Salud en las Américas. Washington: Oficina Regional para las Américas de la Organización Mundial de la Salud; 2020. [citado 2020 jun. 5-10]. Inequidades y barreras en los sistemas de salud. [1 pantalla]. Disponible en: https://www.paho.org/salud-en-las-americas-2017/mhp-inequities-es.html

26. Homedes N, Ugalde A. Las reformas de salud neoliberales en América Latina: una visión crítica a través de dos estudios de caso. Rev. Panam. Salud Pública. 2005; 17(3): 210-220. Diponible en: http:// www.scielosp.org/scielo.php?script $=$ sci_arttext\&pi$\mathrm{d}=$ S1020-49892005000300012\&lng=pt\&nrm=iso\&tl$\mathrm{ng}=\mathrm{es}$

27. Consejo de Organizaciones Internacionales de las Ciencias. International ethical guidelines for biomedical research involving human subject. Ginebra (Suiza): Consejo de Organizaciones Internacionales de las Ciencias Médicas (cioms); 2002. [citado 2020 jun. 15]. Disponible en: https://cioms.ch/publications/product/ international-ethical-guidelines-for-biomedical-research-involving-human-subjects-2/

28. Organización Mundial de la Salud. who guidelines on ethical issues in public health surveillance [Internet]. Ginebra (Suiza): Organización Mundial de la Salud; 2017. [citado 2020 jun. 25]. Disponible en: https://apps. who.int/iris/bitstream/handle/10665/255721/9789241 512657-eng.pdf? sequence $=1$

29. Buchanan DR. Autonomy, paternalism, and justice: ethical priorities in public health. Am J Public Health. [Internet]. 2008 [citado 2020 jul. 5]; 98(1): 15-21. Disponible en: DoI: https://doi.org/10.2105/ AJPH.2007.110361.

30. Klugman CM. Public health principlism. Journal of Health Ethics. [Internet]. 2007 [citado 2020 jul. 10]; 4(1). DoI: https://doi.org/10.18785/ojhe.0401.04

31. Coughlin SS. Ethical issues in epidemiologic research and public health practice. Emerg Themes Epidemiol. [Internet]. 2006 oct. 3 [citado 2020 jul. 5]; 3(16). DOI: https://doi.org/10.1186/1742-7622-3-16

32. Alves-Rippel J, de Medeiros CA, Maluf F. Universal declaration on bioethics and human rights and cns resolution 466/12: a comparative analysis. Revista Bioética. [Internet]. 2016 jul. [citado 2020 jul. 5]; 24(3): 603-12. Disponible en: https://search-ebscohostcom. ezproxy.javeriana.edu.co/login.aspx?direct $=$ true\&d$\mathrm{b}=\mathrm{edb} \& \mathrm{AN}=120513129$ \&lang $=$ es\&site $=$ eds -
33. Au SS, Couillard P, Roze des Ordons A, Fiest KM, Lorenzetti DL, Jette N. Outcomes of ethics consultations in adult icus: A systematic review and meta-analysis. Critical Care Medicine. [Internet]. 2018 [citado 2020 mzo. 5]; 46(5): 799-808. DoI: https://doi. org/10.1097/CCM.0000000000002999.

34. Real de Asúa D, Rodríguez del Pozo P, Fins JJ. El internista como consultor de ética clínica: un antídoto contra «la barbarie del especialismo» en la práctica hospitalaria. Rev Clin Esp. [Internet]. 2018 [citado 2020 oct. 5]; 218(3): 142-8 DoI: https://doi.org/10.1016/j. rce.2017.09.005.

35. Soini S, editor. Public health: ethical issues [Internet]. Copenhague: Nordic Council of Ministers; 2011. [citado 2020 oct. 5]. DoI: https://doi.org/10.6027/TN2011-535

36. Schmidt H. Personal responsibility for health - developments under the German healthcare reform 2007. Eur J Health Law. [Internet]. 2007 [citado 2020 mzo. 5]; 14(3): 241-50. Dor: https://doi.org/10.1163/092902707x240602. PMID: 18229761

37. Jatkauskiené B, Siguté N. Visuomenés Sveikata ir etika: praktikos ir moksliniu Tyrimu raidos diskursas. Tiltai. [Internet]. 2018 [citado 2020 oct. 5]; 81(3):111-25. DoI: https://doi.org/10.15181/tbb.v79i3.1895

38. Organización Panamericana de Salud. Recomendaciones sobre la ética de la prevención, atención y control de la tuberculosis [Internet]. Washington: Oficina Regional para las Américas de la Organización Mundial de la Salud; 2013. [citado 2020 oct. 5]. Disponible en: https://apps.who.int/iris/bitstream/handle/10665/89637/9789275317433_spa.pdf?sequence $=1$

39. World Health Organization. Ethics in epidemics, emergencies and disasters: research, surveillance and patient care [Internet]. Ginebra (Suiza): Organización Mundial de la Salud; 2015. [citado 2020 oct. 5]. Disponible en: https://apps.who.int/iris/bitstream/handle/10665/196326/9789241549349_eng.pdf?sequence $=1$

40. Evans N, Hills K, Levine AC. How should the who Guide Access and Benefit Sharing during infectious disease Outbreaks? ama. [Internet]. 2020 [citado 2020 oct. 5]; 22(1): 28-35. DoI: https://doi.org/10.1001/amajethics. 2020.28

41. Sorokin P, Mariel A, Outomuro D. Health care ethics committes:from great dilemmas to new challenges. Rev. bioét. 2016; 24(1): 91-96. DoI: https://doi. org/10.1590/1983-80422016241110

42. La Torre G, Mannocci A, McCarthy M, Ricciardi W, Bes-Rastrollo M. Investigación en salud pública: ¿hay 
diferencias entre los países del norte, el sur y el este de Europa? Una perspectiva desde las asociaciones nacionales de salud pública. Gac Sanit. [Internet]. 2010 [citado 2020 oct. 5]; 24(3): 1-5. DoI: https://doi. org/10.1016/j.gaceta.2009.09.019,

43. Resolución 13437 de 1991 [Ministerio de Salud]. Por la cual se constituyen los comités de ética hospitalaria y se adopta el decálogo de los derechos de los pacientes. Noviembre 10 de 1991.

44. Decreto 1757 de 1994 [Ministerio de Salud]. Por el cual se organizan y se establecen las modalidades y formas de participación social en la prestación de servicios de salud, conforme a lo dispuesto en el numeral 1 del artículo 4 del decreto-ley 1298 de 1994. Agosto 3 de 1994.

45. Ley 1164 del 2007. Por la cual se dictan las disposiciones en materia de talento humano en salud. Octubre 3 de 2007. DoI: https://doi.org/46.771

46. Carrillo-González S. Situación actual de los comités de bioética clínico-asistenciales en la ciudad de Cartagena. Pers y bioética. [Internet]. 2013 [citado 2020 oct. 5];17(1): 96-110. Disponible en: https://www.redalyc. org/pdf/832/83228613008.pdf

47. Decreto 1954 de 2019 [Departamento administrativo de Ciencia, Tecnología e Innovación]. Por el cual se de- signan los miembros del Consejo Nacional de Bioética. Octubre 25 de 2019.

48. Rueda Martínez G, Monsores de Sá N. Impact of the absence of the Colombian national council of Bioethics. [Internet]. Rev latinoam bioet. 2015; 15(2): 144155. Disponible en: rev.latinoam.bioet.

49. Upshur R. Principles for the justification of public health intervention. Can J Public Health. [Internet]. 2002 mzo. [citado 2020 oct. 5]; 93(2):101-3. Disponible en: https://www.researchgate.net/publication/11400293

50. Roth AN. Políticas públicas, formulación, implementación y evaluación. 13. a ed. Bogotá: Ediciones Aurora; 2018.

51. Buitrago-Ramírez F, Ramón CM, Fernández-Alonso M, Tizón JL. Pandemia de la covid-19 y salud mental: reflexiones iniciales desde la atención primaria de salud española. Atención Primaria. [Internet]. 2021 [citado 2020 oct. 5]; 53(1): 89-101. Disponible en: DoI: https://doi.org/10.1016/j.aprim.2020.06.006

52. Nussbaum MC. La monarquía del miedo: una mirada filosófica a la crisis política actual. Barcelona: Ediciones Paidós; 2019. 